\title{
BMJ Open Improving care standards for patients with spinal trauma combining a modified e-Delphi process and stakeholder interviews: a study protocol
}

\author{
Lisa N Sharwood, ${ }^{1,2}$ Ralph Stanford, ${ }^{3}$ James W Middleton, ${ }^{1,2,4}$ Brian Burns, ${ }^{1,5}$ \\ Anthony Joseph, ${ }^{6}$ Oliver Flower, ${ }^{6}$ Oran Rigby, ${ }^{2,5,7}$ Jonathon Ball, ${ }^{2,6}$ Shelly Dhaliwal ${ }^{2}$
}

To cite: Sharwood LN, Stanford R, Middleton JW, et al. Improving care standards for patients with spinal trauma combining a modified e-Delphi process and stakeholder interviews: a study protocol. BMJ Open 2017:7:e012377.

doi:10.1136/bmjopen-2016012377

- Prepublication history for this paper is available online To view these files please visit the journal online (http://dx.doi.org/10.1136/ bmjopen-2016-012377).

Received 21 April 2016 Revised 31 October 2016 Accepted 22 December 2016

CrossMark

For numbered affiliations see end of article.

Correspondence to Dr Lisa N Sharwood; lisa.sharwood@sydney.edu.au

\section{ABSTRACT}

Introduction: Around 300 people sustain a new traumatic spinal cord injury (TSCl) in Australia each year; a relatively low incidence injury with extremely high long-term associated costs. Care standards are inconsistent nationally, lacking in consensus across important components of care such as prehospital spinal immobilisation, timing of surgery and timeliness of transfer to specialist services. This study aims to develop 'expertly defined' and agreed standards of care across the majority of disciplines involved for these patients.

Methods and analysis: A modified e-Delphi process will be used to gain consensus for best practice across specific clinical early care areas for the patient with TSCI; invited participants will include clinicians across Australia with relevant and significant expertise. A rapid literature review will identify available evidence, including any current guidelines from 2005 to 2015 . Level and strength of evidence identified, including areas of contention, will be used to formulate the first round survey questions and statements. Participants will undertake 2-3 online survey rounds, responding anonymously to questionnaires regarding care practices and indicating their agreement or otherwise with practice standard statements. Relevant key stakeholders, including patients, will also be interviewed face to face.

Ethics and dissemination: Ethics approval for this study was obtained by the NSW Population \& Health Services Research Ethics Committee on 14 January 2016 (HREC/12/CIPHS/74). Seeking comprehensive understanding of how the variation in early care pathways and treatment can be addressed to achieve optimal patient outcomes and economic costs; the overall aim is the agreement to a consistent approach to the triage, treatment, transport and definitive care of acute TSCI victims. The agreed practice standards of care will inform the development of a Clinical Pathway with practice change strategies for implementation. These standards will offer a benchmark for state-wide and potentially national policy.

\section{Strengths and limitations of this study}

- This study will obtain expert consensus on specific agreed practice standards and associated 'best practice' models of care in the acute postinjury period for the patient with traumatic spinal cord injury. Previous studies have demonstrated lack of consensus, particularly in this phase of care.

- Conducting an online survey will permit the range of experts invited, to respond anonymously; the sample of invited participants across Australia has been carefully selected and the consensus of their opinions will produce a respected set of standards.

- The views of respondents to the Delphi survey may differ from those experts who choose not to participate, and these clinicians may resist resultant drive to change policy and practice.

\section{INTRODUCTION}

Defining 'agreed standards for practice' is one of the first and most crucial steps in the translation of knowledge into policy and practice. Without an agreed, evidence-based benchmark, identifying gaps in current practice cannot occur. ${ }^{1}$ Agreed practice standards are able to inform and enable the development and implementation of new models of care. However, in order to successfully change practice, it is vital to fully understand potential barriers to implementation of defined standards. Key informants to this process in the acute healthcare setting include stakeholders such as the prehospital and acute care clinicians (ie, ambulance officers, trauma doctors and nurses, emergency physicians, neuroradiologists, orthopaedic and neurosurgeons), policymakers, healthcare service managers and the patients themselves. 
Around 300 people sustain a new traumatic spinal cord injury (TSCI) in Australia each year, with an estimated 15 new cases per million population each year (aged $>15$ years), ${ }^{2}$ yet despite a relatively low incidence, the long-term costs associated with TSCI are extremely high. The lifetime financial cost has previously been estimated at $\$ 5$ million for a person with paraplegia and $\$ 9.5$ million for quadriplegia; ${ }^{3}$ in addition to this, there are substantial human and social costs. It is well documented that the early phase of care can significantly impact long-term outcomes for the patient with TSCI; ${ }^{45}$ however, while there has been some improvement in referral patterns to specialist centres, there remains significant variation in policy and practice among what is considered 'best practice' specialist care.

A pilot study ${ }^{6}$ retrospectively linked prehospital and hospital outcome data for a group of patients with TSCI between 2004 and 2008 in New South Wales (NSW), Australia. Analysis informed that patients injured in nonmetropolitan and rural regions reached specialist care (in a Spinal Cord Injury Unit-SCIU) in Sydney faster than those transported initially by ambulance to a major (non-SCIU) metropolitan trauma service. Patients who had sustained multitrauma were more likely to experience delays than those with isolated TSCI; however, delays of more than 24 hours to reach an SCIU were also shown in patients with isolated TSCI. Secondary complications such as deep vein thrombosis (DVT) or pressure injury were experienced with 2.5 times greater likelihood in patients who experienced delays $(95 \%$ CI 1.51 to $4.17, \mathrm{p}<0.001) .{ }^{6}$ Barr $^{7}$ also reported significantly increased complication risk on admission with such delays, and additionally, significantly longer lengths of hospital stay for patients with complications on admission. Hospital length of stay has been demonstrably reduced by $30 \%$ with early admission to an SCIU, this practice also achieving a threefold reduction in the rate of pressure ulcers ${ }^{8}$ as well as a reduced DVT incidence $(2 \%$ vs $26 \%) .^{9}$ In addition, several recent studies employing state population-based linked administrative data sets in NSW (Sharwood L, Boufous S, Muecke S, et al. Linked health record data demonstrates inconsistency and delays in care pathways for patient with traumatic spinal cord injury. Injury 2016. under resubmission review) and Canada (Ontario) ${ }^{10}$ have identified patient-specific and health system factors that may pose barriers to expeditious spinal cord injury care, such as increased age, number of interhospital transfers and thoracolumbar injuries.

Ideal treatment of the patient with suspected TSCI depends on having an effective and coordinated healthcare system capable of recognising and treating this injury as a medical emergency, employing spinal precautions then rapidly and directly transporting them to an SCIU. ${ }^{4}$ Guidelines for the early management of acute spinal cord injuries have been developed by the Consortium for Spinal Cord Medicine ${ }^{11}$ and more recently the Congress of Neurological Surgeons; ${ }^{12}$ however, anecdotal evidence suggests these are not followed uniformly, nor agreed on and applied consistently. Currently, there are limited population-level data on the outcomes, size and nature of the effect of delayed initiation of specialist care in SCIU, and the role other factors play in the early care period after TSCI onset. Data are also limited about the journey of a patient with suspected TSCI from the time of injury to definitive care; the specific practices and processes that cause delay and the extent to which these factors are amenable to modification.

The proposed project will (1) obtain expert consensus on specific agreed practice standards and associated 'best practice' models of care in the acute postinjury period for the patient with TSCI using existing guidelines and updated evidence, and (2) identify drivers (barriers and facilitators) of 'best practice', namely clinician, organisational or policy factors.

Further to this, and in the next phase of a larger suite of projects, the findings from these steps will enable the measurement of current practice against the agreed practice standards to describe the evidence-practice gap. This will be performed using data from a completed prospective observation study. ${ }^{13}$ The overall aim, beyond this Delphi study, is to enable the publishing of these agreed standards as a practice benchmark and facilitate their translation into NSW state-wide policy, with indicators to monitor health system performance.

\section{METHODS AND ANALYSIS}

\section{Rapid review}

Rapid review is becoming an increasingly favoured methodology $^{14}$ to facilitate the appraisal and synthesis of evidence in a time efficient manner, especially when informing healthcare policy and practice decisions, with this technique recently reported in SCI. ${ }^{15}$ The authors have chosen a rapid review to identify the existing guidelines and to update evidence from latest literature reporting clinical research and systematic reviews. The primary focus of this Delphi consultative process is to obtain consensus about a best practice model in the key areas of prehospital, early hospital, diagnostic imaging and haemodynamic management. A steering committee will also share their own knowledge, including information on existing policies, protocols and guidelines currently in place for the prehospital and early hospital care of TSCI patients; all of which will contribute to the Delphi survey questions and consensus statements for development.

The authors will prepare rapid review evidence for four topics specific to the acute phase of care for the patient with TSCI, namely (1) prehospital care, including triage and transfer protocols and spinal precautions during transport; (2) early hospital care, including spinal stabilisation, neurological and multitrauma assessment, (3) diagnostic imaging and (4) haemodynamic management. The rapid review search strategy will be 
limited to years of publication 2005-2015, English language and humans aged 16 years or more.

Any new evidence not considered in the formulation of the most recent guideline recommendations ${ }^{12}$ will be rated using a modification of the North American Spine Society criteria for evaluation of the medical literature $^{16}$ to maintain consistency in rating the strength of identified evidence, described by Walters, ${ }^{17}$ and used by the Association of Neurological Surgeons and Congress of Neurological Surgeons in recent guidelines. This incorporated three classes of evidence; Class 1 includes randomised controlled trial (RCT) or systematic reviews of these, Class 2 case-control studies, prospective or retrospective comparative studies, lesser quality RCTs or systematic reviews of any of these as well as Class 1 studies with inconsistent results and Class 3 incorporates case series or reports and expert opinion. Identified guidelines will be examined using the AGREE Reporting Checklist; ${ }^{18}$ the same tool will be applied in any guideline developed as a result of this study. In the absence of current, published reporting standards for rapid reviews, the PRISMA statement ${ }^{19}$ will be used, where applicable, to report the rapid review. Search strategies used will be appended to research finding publications.

\section{The Delphi process}

A modified e-Delphi process (with closed first round informed by rapid review) will be used for consensus building about 'best practice', following a structured communication process whereby opinions are collected and summarised from content experts and through the facilitated and iterative process, individual expert opinions are synthesised into an aggregated view. Online consensus rounds (Delphi surveys) for participants will be conducted to identify key, time-sensitive aspects of an integrated clinical pathway and specialised care. Surveys will be sent electronically with a formal letter of invitation that contains the ethically approved Participant Information Statement. Completion and return of the survey will be taken as participant consent. Interim, anonymous feedback from the facilitator encourages anonymous revision of responses where needed; the process is finished after a predefined stop criterion and mean/median scores can be reported. It is believed that during this process, the range of the answers will converge towards a 'right' answer. ${ }^{20}$ The Delphi process facilitates inclusivity and confidentiality; and is equally inclusive regardless of a participant's geographic or administrative location. In this context, 'best practice' is defined as evidence-based, high-quality policy, embedded in a well-organised structure with effective communication to facilitate consistent outcomes.

In order to define best practice for prehospital and inhospital acute phases of care; survey participants will comprise a diverse, multidisciplinary panel of clinicians (medical, nursing, radiology, surgery), academics, researchers, healthcare decision-makers (eg, Agency for
Clinical Innovation, NSW) nationally, with expertise and experience in acute TSCI care.

\section{The Delphi survey}

Study data will be collected and managed using REDCap electronic data capture tools hosted at The University of Sydney. REDCap ${ }^{21}$ (Research Electronic Data Capture) is a secure, web-based application designed to support data capture for research studies, providing: (1) an intuitive interface for validated data entry; (2) audit trails for tracking data manipulation and export procedures; (3) automated export procedures for seamless data downloads to common statistical packages; and (4) procedures for importing data from external sources. Participants to be invited to complete the survey will have been identified as 'content experts' by the steering committee, comprising a national representation of prehospital and acute care clinicians, including ambulance officers, trauma doctors and nurses, emergency physicians, neuroradiologists, orthopaedic and neurosurgeons. Invited participants will be sent a link to the online survey. Two to three online survey rounds will be conducted for participants, $\sim 6$ weeks apart. The first part of the survey is designed to document current practice and provide epidemiological information such as professional background, including number of years in service. The second part of the survey contains the Delphi statements, to which participants will indicate their level of agreement. Review of responses from Round 1 will permit modification, removal or reformulation of a statement, aiming for eventual collation of views on a particular topic. An item will remain in the subsequent round where its level of agreement in the first was $80 \%$. The survey questions will come from the four main topic areas outlined above, considering also care protocol-related subtopics such as skin pressure management, timing of decompressive surgery and complication prevention. The clinician's view on the timing of surgery can influence the patient's early care leading up to surgery. Questions will either be a practice statement that demands either an affirmative or negative response, or a statement regarding standards where several options may be checked. There will be an option for text comments at the end of each topic area, to offer clarification or further information.

At the beginning of each survey, participants will be requested to provide a brief overview of their current practice, which will be used to inform an understanding of variation across different settings nationally. In order to prevent attrition bias, all participants will be offered a \$100 David Jones () voucher, in return for their time and completion of the survey. Up to four reminder emails will be sent at 7-day intervals from the initial email invitation. Participants completing Round 1 will be further invited to complete Round 2. Summary results from Round 1 will be provided to Round 2 participants by way of opening the next round. The same procedure will be followed should a Round 3 be required. 
The authors acknowledge that some participants may not have expertise in all four areas; therefore, while all participants will be encouraged to respond to all questions within the four main Delphi topics, some may choose to limit their responses to their own area of expertise only.

\section{Stakeholder interviews: TSCI clinicians and patients}

In order to gain a full understanding of the individual practitioner, organisational, policy, resource or other diverse factors perceived to influence practice and patient flow, key stakeholder insights and experiences will be captured through a series of semistructured face-to-face interviews with relevant staff across services in NSW. This will include purposive sampling of senior paramedics, medical, surgical and nursing staff at regional and major trauma services and the specialist SCIUs, as well as relevant hospital managers. Interview respondents will be asked to provide answers to questions that relate to their remit, for example, senior paramedic staff will only be asked to respond to questions regarding care of the patient with TSCI from the scene of injury to the destination hospital, whereas hospital managers will be asked to respond to questions regarding acceptance of patients with TSCI under their service, non-refusal policies, etc. They will be invited by electronic mail, and followed up by telephone contact to obtain their consent. The face-to-face interviews will be conducted at a time convenient to the research team and the respondent. With respect to sample size, ethics approval is for $\sim 10$ respondents per clinical practice area and no more than 10 patients.

In addition, a discrete selection of patients who have sustained a TSCI in the past 12 months will also be invited to participate in 'in-depth' interviews (with informed consent) to understand the consumer experience of healthcare received along the clinical pathway. Inpatients at the two specialist spinal cord injury services in NSW will be approached to obtain face-to-face opt-in consent at the point of interview. Patients will only be selected following discussion with the medical and nursing directors of each service, to determine appropriateness. The research assistant will explain the study objectives to them and leave behind the consent form, which they can sign without any coercion should they choose to participate in the study.

The objective is to explore current practice within the context of the agreed standards of care and clinical pathway/s, and importantly, to identify factors perceived as barriers or facilitators (drivers) of improved practice. Face-to-face interviews aim is to elicit the extent to which clinician knowledge, skills, attitudes and behaviours, as well as local policy, management structure and resources, and other organisational factors, such as interdisciplinary communication, influence the care of the patient with TSCI. More specifically, characteristics of facilitators will be identified, such as education, communication mechanisms or interservice transfer policies and processes that provide keys for system-wide strategic improvement. The clear purpose of the interview will be established at the outset; that the ultimate goal will be improved patient outcomes. Respondents will be asked to describe their experiences of barriers and facilitators to achieving best practice care for patients with acute TSCI in their care.

All interviews will be audio taped with consent, transcribed, then returned to interviewees for review, correction or addition of points where necessary, prior to being finalised for analysis.

\section{Steering committee}

Project oversight will be performed by a steering committee of eight content knowledge experts, led by the project investigator. These experts will include prehospital and retrieval specialists, emergency care professionals, trauma directors and clinicians, intensivists, spinal surgeons and specialist physicians and clinician researchers across the key areas to be covered in the Delphi survey.

The steering committee will oversee the entire process of (1) reviewing and assessing the level of evidence, as well as knowledge gaps in the literature identified by rapid review, (2) informing development of the first round questions and statements for modified Delphi survey, (3) analysing the results to ensure the progression between rounds clearly establishes consensus of at least $80 \%$, refining survey questions where necessary, (4) exploring themes identified in key stakeholder interviews as evidence practice gaps in service-delivery and practice and (5) recommending feasible strategies that address these gaps in the acute phase of care of patients with TSCI.

\section{Analysis and reporting}

Statistical analysis of the Delphi survey findings will predominantly report measures of central tendency (means, median and mode) and level of dispersion (SD and interquartile range) in order to present information concerning the collective judgements of respondents. Final decisions on standards to include as the 'best practice' components of care will be determined by a consensus of $>80 \%$.

\section{Qualitative content analysis}

The transcribed indepth interview data (from clinician, patient and other stakeholder interviews) will undergo qualitative content analysis (Mayring P. Qualitative content analysis: theoretical foundation, basic procedures and software solution. In: SSOAR, ed. Klagenfurt: www.ssoar.info, 2014:144), ${ }^{22}$ by means of the QCAmap software (http://www.qcamap.org), using the methods according to Mayring (Mayring P. Qualitative content analysis: theoretical foundation, basic procedures and software solution. 2014). Using inductive reasoning, this will involve identification of the scope and context of key themes, followed by assignment of codes to data with 
similar characteristics. A set of coding rules consisting of definitions of anticipated technical terms will be defined 'apriori' by the Steering Committee, as recommended by Potter and Levine-Donnerstein. ${ }^{22}$ Clustering descriptive codes into groups of related key concepts will permit analysis by thematic coding, linking findings to the quantitative outcomes. ${ }^{23}$ Supportive quotes will be identified and described, where intercoder reliability demonstrates agreement of selected quotes. Creation of connections between the data sets should enable a deeper level of analysis and interpretation than would be possible using only one or the other. While it is possible for this type of concurrent and multilevel analysis to deduce dissimilar findings, creating difficulties with triangulation or cross validation of findings, structured approaches to dealing with discrepancies should maximise understanding and augment the strength of the findings. Two researchers (LNS, SD) will undertake the qualitative analysis, creating categories or themes based on valid interpretation to then produce group codes. ${ }^{23}$ A coding framework will be created to ensure intercoder reliability and coding consistency. Validity will be ensured through the creation of a coding manual which will be adjoined in publication of the findings, in which researcher bias will be considered and acknowledged. The COREQ checklist will be used for the reporting of the qualitative study. ${ }^{24}$

Additional software used in the facilitation and conduct of this project will include Microsoft Share point, Office 365 , REDCap $^{21}$ and SPPS.

\section{Dissemination}

This study forms an additional part of a large observation study of patients with TSCI. ${ }^{13}$

Study findings will be considered in the light of current policy and practice, with scrutiny of evidence to identify specific improvements to be advised in relation to practice. Any recommendations as to the essential components and timing of 'specialist spinal care' will be formulated into a Best Practice Clinical Pathway, formulated using the internationally accepted standards of the AGREE instrument. ${ }^{18}$ Descriptions of the process indicators and performance measures feasible for state-wide system benchmarking, and aiming to improve patient outcomes will be prepared in a report to be formalised in proposal to relevant stakeholders such as the NSW Ministry for Health or the Institute for Trauma and Injury Management, inviting engagement towards implementing change where a 'business case' can be made. Research findings will be published in academic literature, using media engagement to disseminate significant findings.

Identification of specific barriers to best practice through the range of key stakeholder interviews will facilitate development of targeted improvement strategies, aiming for greater efficiency of our services, focused resource usage, highest standard of practice and consequently, optimal outcomes. This project is ideally placed to establish standards and inform the development of a Clinical Pathway with practice change strategies for implementation offering best practice systems across our healthcare service in NSW. Emphasis will be placed on developing a comprehensive understanding of how the variation in early care pathway/s and treatment can be addressed to achieve optimal patient outcomes and reduction in economic costs to the community; the overall aim being to see a consistent approach to the triage, treatment, transport and definitive care of TSCI victims. This comprehensive approach aims to address national health priority issues, and equally learn from, and provide learning to other national and international performance and policy models.

\section{CONCLUSION}

The long-term impact of TSCI on our healthcare system is significant and ongoing. Deficits in clinical policy and practice can adversely affect neurological outcomes and recovery ${ }^{25}$ and escalate personal and economic costs for the patient, as well as the healthcare system. The proposed project outlines a plan to employ a modified e-Delphi process to define the evidence-based best practices for implementation and identify the associated performance measures and indicators to evaluate current practice in acute TSCI care. The global objective is to improve compliance with specific clinical processes, importantly reducing practice variation, ideally leading to the implementation of an agreed model of best practice on a national scale, using this quality improvement process in national benchmarking.

The final component of the Delphi Process (as a next phase project) will therefore be to clearly specify the essential components of 'specialist spinal care' to include in a Clinical Pathway, describing performance measures suitable for system-wide benchmarking and improving patient outcomes. This will help to define optimal timing and care components along the clinical pathway, and performance measures aiming to minimise treatment delays and optimise outcomes in the Australian healthcare system context.

Author affiliations

${ }^{1}$ Sydney Medical School Northern, The University of Sydney, Sydney, New South Wales, Australia

2John Walsh Centre for Rehabilitation Research, Kolling Institute, Northern Sydney Local Health District, Sydney, New South Wales, Australia

${ }^{3}$ Prince of Wales Hospital, Sydney, New South Wales, Australia

${ }^{4}$ State Spinal Cord Injury Service, NSW Agency for Clinical Innovation, Sydney, New South Wales, Australia

${ }^{5}$ Greater Sydney Area Helicopter Emergency Medical Service, Sydney, New South Wales, Australia

${ }^{6}$ Royal North Shore Hospital, Sydney, New South Wales, Australia

${ }^{7}$ Institute for Trauma and Injury Management, NSW Agency for Clinical Innovation, Sydney, New South Wales, Australia

Contributors LNS conceived and designed the study with the support of JM. LNS drafted the manuscript and incorporated the revisions between authors. SD edited all versions prior to final versions. All authors read and approved the final manuscript. 
Funding This study is funded by a Sydney Medical School Early Career Researcher Grant awarded to LNS (2015-2016).

Competing interests LNS and SD had financial support from Sydney Medical School for the submitted work.

Ethics approval NSW Population \& Health Services Research Ethics Committee.

Provenance and peer review Not commissioned; externally peer reviewed.

Data sharing statement This is a study protocol.

Open Access This is an Open Access article distributed in accordance with the Creative Commons Attribution Non Commercial (CC BY-NC 4.0) license, which permits others to distribute, remix, adapt, build upon this work noncommercially, and license their derivative works on different terms, provided the original work is properly cited and the use is non-commercial. See: http:// creativecommons.org/licenses/by-nc/4.0/

\section{REFERENCES}

1. Bosch M, Tavender E, Bragge P, et al. How to define 'best practice' for use in Knowledge Translation research. J Eval Clin Pract 2013;19:763-8.

2. Norton L. Spinal cord injury, Australia 2007-2008. Injury research and statistics series no. 52. Cat. no. INJCAT 128. Canberra: Australian Institute of Health and Welfare 2010.

3. Access Economics Pty Ltd. The economic cost of spinal cord injury and traumatic brain injury in Australia. Melbourne: VNI, 2009:1-116.

4. Ahn H, Singh J, Nathens A, et al. Prehospital care management of a potential spinal cord injured patient: a systematic review. J Neurotrauma 2011;28:1341-61.

5. Cone D, Irvine K, Middleton P. Australian Prehospital Outcomes Study of Longitudinal Epidemiology (APOSTLE) project. Prehospital Emerg Care 2012;16:505-12.

6. Middleton PM, Davies SR, Anand S, et al. The prehospital epidemiology and management of spinal cord injuries in NSW: 2004-2008. Injury 2012;43:480-5.

7. Barr F. Preserving \& Developing the National Spinal Cord Injury Service. London: Association SI, 2009:1-32.

8. Ploumis A, Kolli S, Patrick M, et al. Length of stay and medical stability for spinal cord-injured patients on admission to an inpatient rehabilitation hospital: a comparison between a model SCI trauma center and non-SCI trauma center. Spinal Cord 2011;49:411-15.

9. Aito S, Pieri A, D'Andrea M, et al. Primary prevention of deep venous thrombosis and pulmonary embolism in acute spinal cord injured patients. Spinal Cord 2002;40:300-3.

10. Wilson J, Voth J, Singh A, et al. Defining the pathway to definitive care and surgical decompression after traumatic spinal cord injury: results of a Canadian population-based cohort study. J Neurotrauma 2016;33:963-71.

11. Consortium for Spinal Cord Medicine. Early acute management in adults with spinal cord injury: a clinical practice guideline for healthcare professionals. J Spinal Cord Med 2008;31:403-79.

12. Walters BC, Hadley MN, Hurlbert RJ, et al. Guidelines for the management of acute cervical spine and spinal cord injuries: 2013 update. Neurosurgery 2013;60(Suppl 1):82-91.

13. Middleton JM, Sharwood LN, Cameron $\mathrm{P}$, et al. Right care, right time, right place: improving outcomes for people with spinal cord injury through early access to intervention and improved access to specialised care: study protocol. BMC Health Serv Res 2014;14:600.

14. Khangura S, Konnyu K, Cushman R, et al. Evidence summaries: the evolution of a rapid review approach. Syst Rev 2012;1:10.

15. Bragge P, Piccenna L, Middleton J, et al. Developing a Spinal Cord Injury Research Strategy using a structured process of evidence review and stakeholder dialogue. Part I: Rapid review of $\mathrm{SCl}$ prioritisation literature. Spinal Cord 2015;53:714-20.

16. NGC. Guideline summary: diagnosis and treatment of degenerative lumbar spinal stenosis: rating scheme for the strength of the evidence. In: National Guideline Clearinghouse (NGC). North American Spine Society, ed. Rockville, MD: Agency for Healthcare Research and Quality (AHRQ), 2011:5-7.

17. Walters $B$. Methodology of the guidelines for the management of acute cervical spine and spinal cord injuries. Neurosurgery 2013;72 (Suppl 2):17-21.

18. Brouwers M, Kerkvliet K, Spithoff K, et al. The AGREE Reporting Checklist: a tool to improve reporting of clinical practice guidelines. BMJ 2016;2016:i1152.

19. Moher D, Liberati A, Tetzlaff J, et al. Preferred Reporting Items for Systematic Reviews and Meta-Analyses: the PRISMA Statement. PLoS Med 2009;6:e10000097.

20. Hasson F, Keeney S, McKenna $\mathrm{H}$. Research guidelines for the Delphi survey technique. J Adv Nursing 2000;32:1008-15.

21. Harris PA, Taylor R, Thielke R, et al. Research electronic data capture (REDCap) - a metadata-driven methodology and workflow process for providing translational research informatics support. $J$ Biomed Inform 2009;42:377-81.

22. Potter W, Levine-Donnerstein D. Rethinking validity and reliability in content analysis. J Appl Commun Res 1999;27:258-84.

23. Zhang $Y$, Wildemuth $B$. Qualitative analysis of content. In: Wildemuth $\mathrm{B}$, Santa Barbara C, eds. Applications of social research methods to questions in information and library. Greenwood Press, 2009:308-19.

24. Tong A, Sainsbury P, Craig J. Consolidated criteria for reporting qualitative research (COREQ): a 32-item checklist for interviews and focus groups. Int J Qual Health Care 2007;19:349-57.

25. Todd NV, Skinner D, Wilson-MacDonald J. Secondary neurological deterioration in traumatic spinal injury: data from medicolegal cases. Bone Joint J 2015;97-B:527-31. 